\title{
A4 Paper Chemistry: Synthesis of a Versatile and Chemically Modifiable Cellulose Membrane
}

\author{
Eungjin Ahn, ${ }^{1,2} *$ Taehyung Kim, ${ }^{1,3}$ Yuju Jeon, ${ }^{3}$ and Byeong-Su Kim ${ }^{1} *$ \\ ${ }^{1}$ Department of Chemistry, Yonsei University, Seoul 03722, Republic of Korea \\ ${ }^{2}$ Department of Biological Chemistry, University of Michigan, Ann Arbor, Michigan 48109, \\ United States \\ ${ }^{3}$ Department of Energy Engineering, Ulsan National Institute of Science and Technology \\ (UNIST), Ulsan 44919, Republic of Korea
}

E-mail: bskim19@yonsei.ac.kr; eungjina@umich.edu 
(a) Energy consumption 1965 - 2015

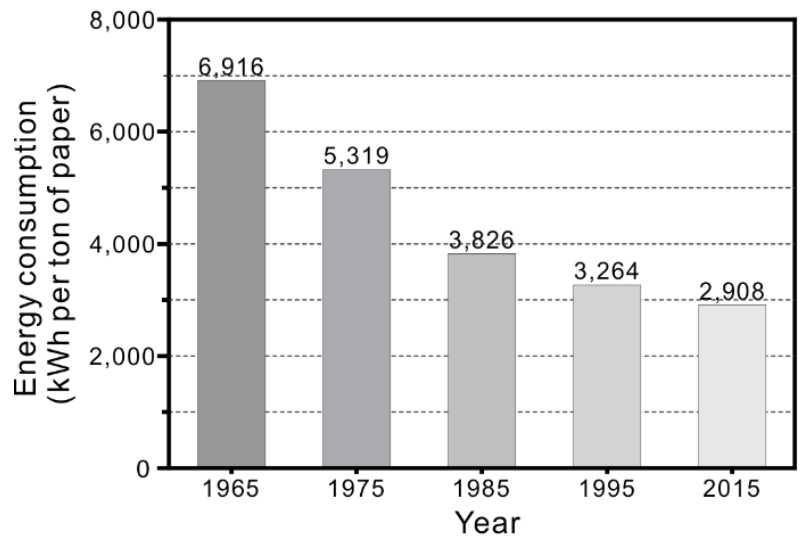

(b) Global paper production $1965-2015$

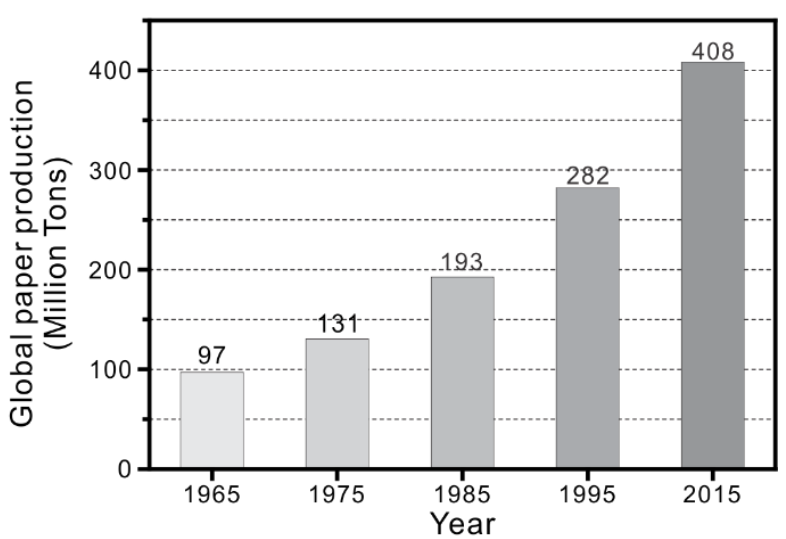

(c)

Distribution of paper production worldwide in 2017

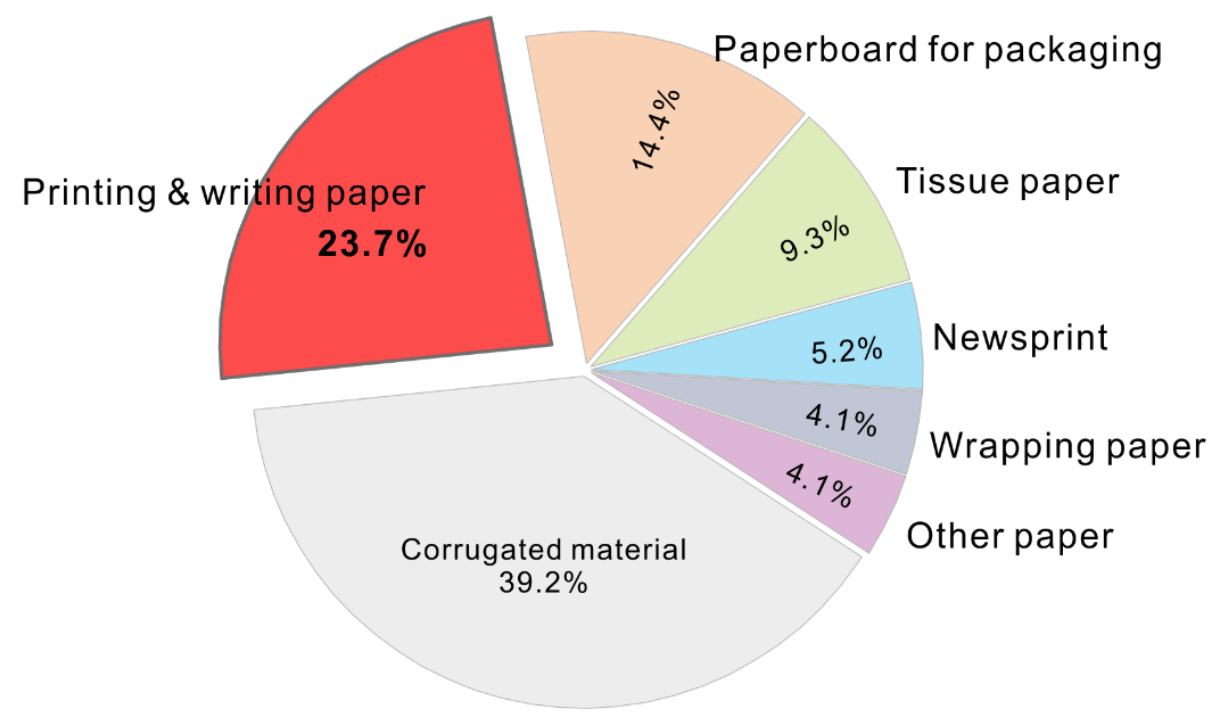

Figure S1. (a) Energy inputs for paper production from 1965 to 2015 in kWh per ton of paper. ${ }^{1}$ (b) Global production capacity of paper and paperboard from 1965 to 2015 in million tons. ${ }^{2}$ (c) Distribution of global paper production in 2017 by grade. ${ }^{3}$ Images were reproduced from statistics with permissions by FAO and Statista. 
(a)

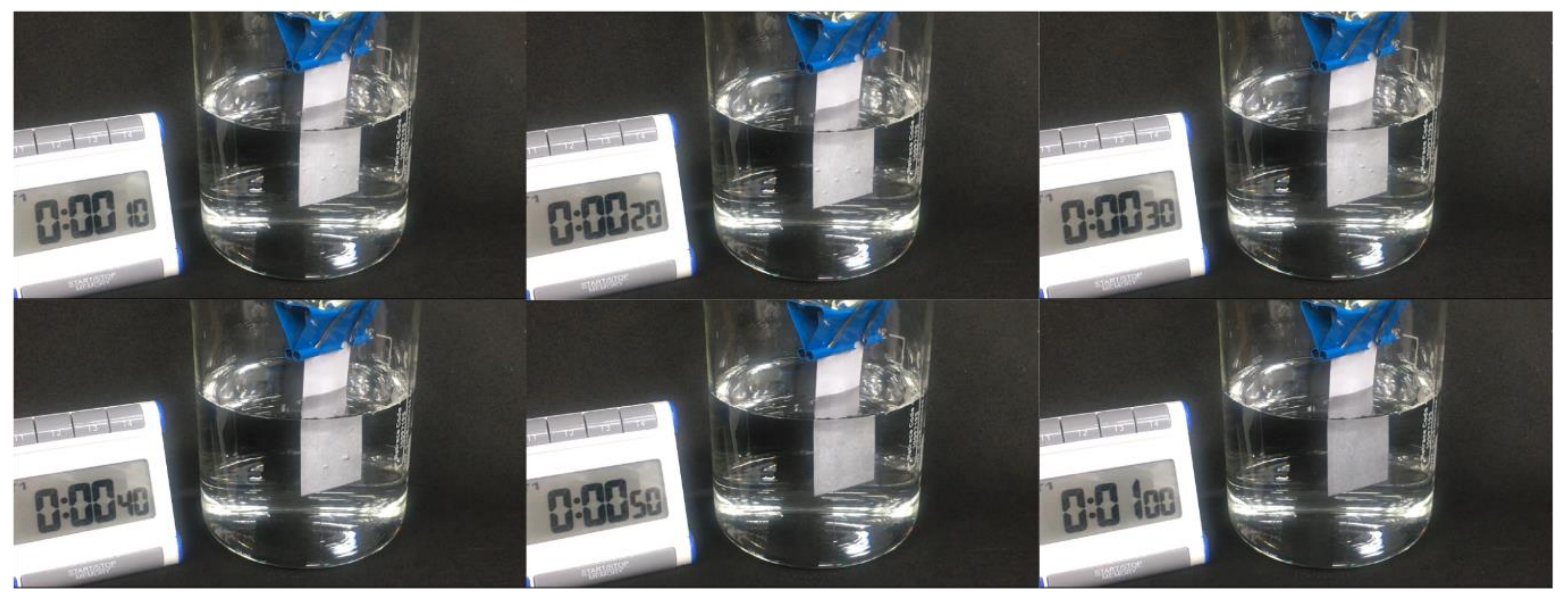

(b)

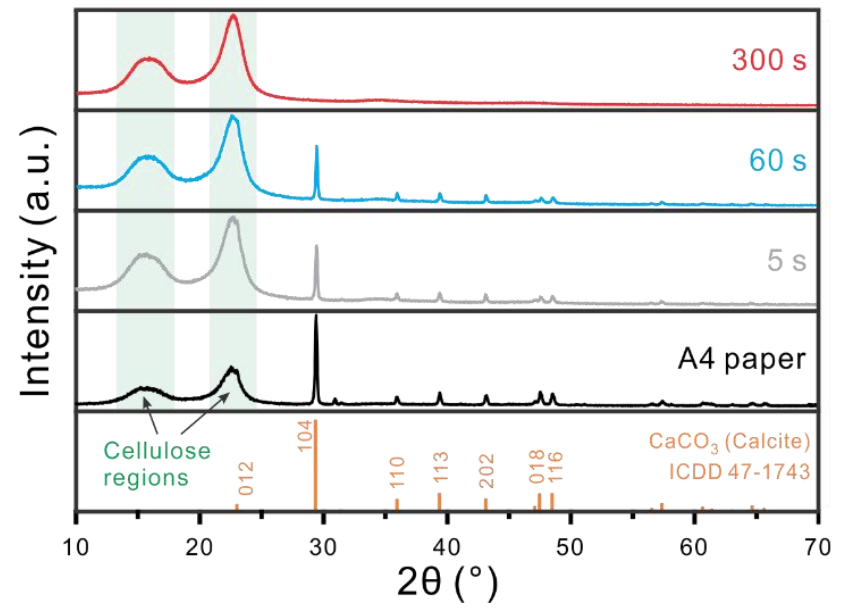

(c)

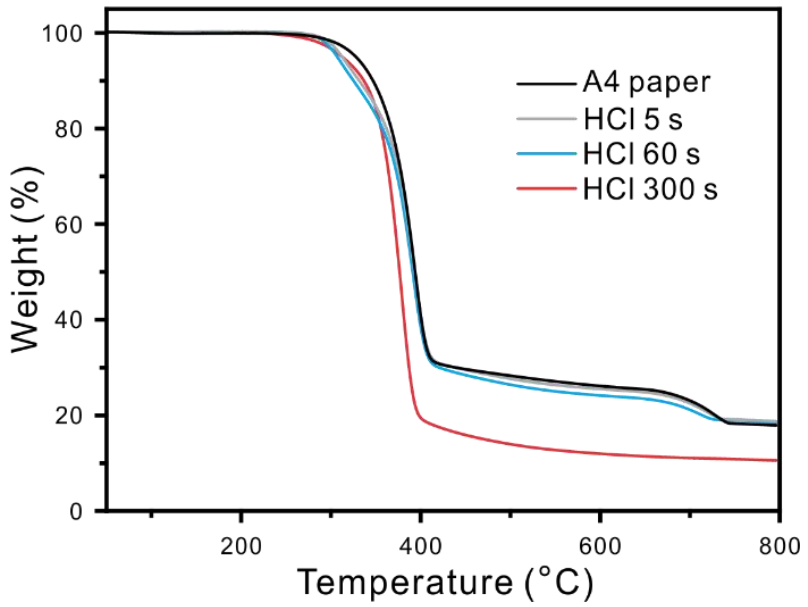

Figure S2. (a) Generation of $\mathrm{CO}_{2}$ bubbles on the surface of commercial $\mathrm{A} 4$ paper after dipping in $\mathrm{HCl}$ solution for $1 \mathrm{~min}$. (b) XRD and (c) TGA analysis of acid-treated papers with $200 \mathrm{mM} \mathrm{HCl}$ solution under different treatment times. 
Supporting Information

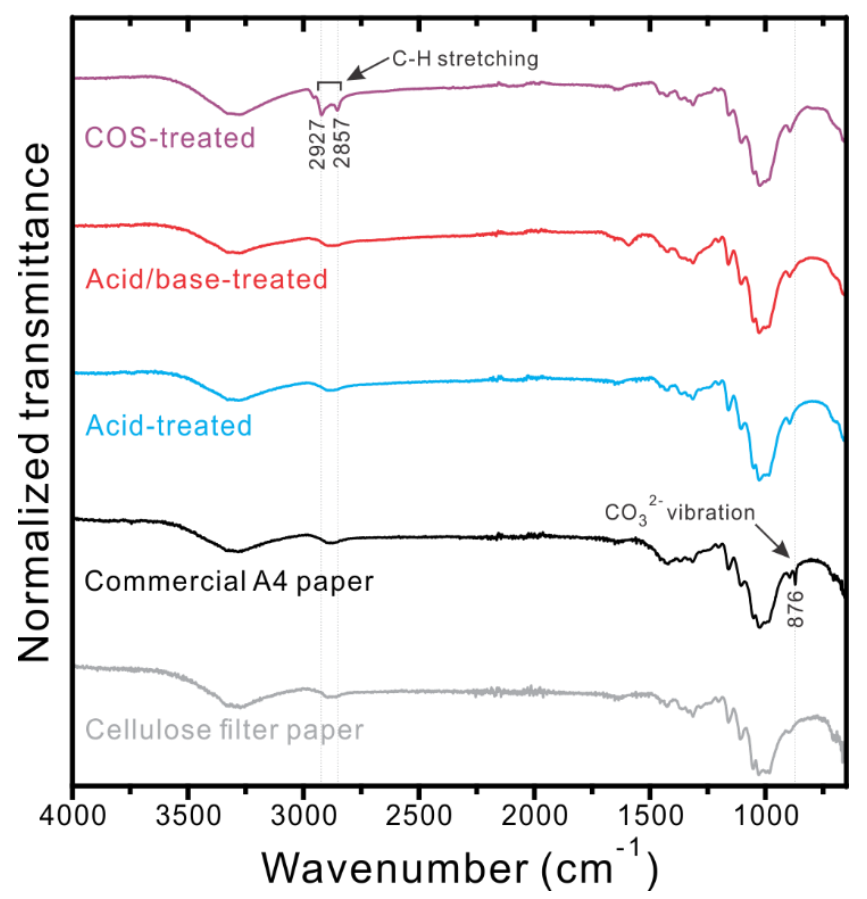

Figure S3. FT-IR spectra of A4 paper modified membranes and commercial cellulose filter paper. 


\section{COS-treated membrane after water rinse}

(a)

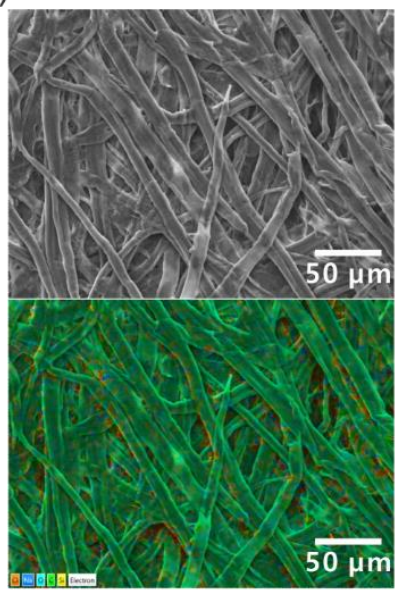

(b)

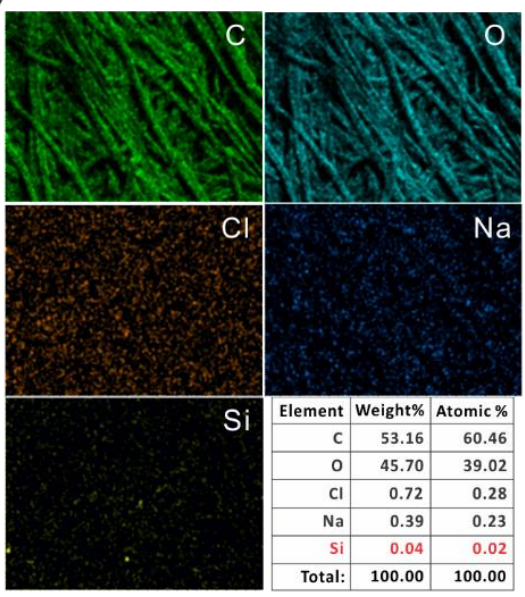

(c)

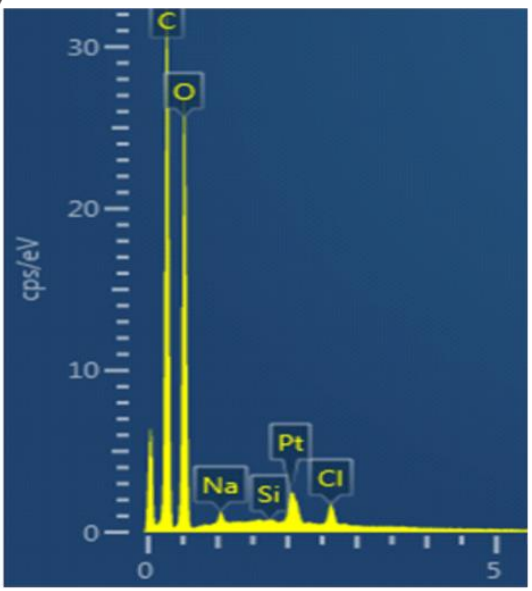

\section{COS-treated membrane after hexane rinse}

(d)

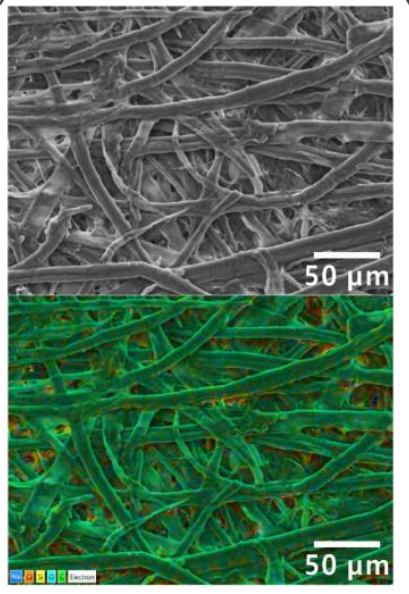

(e)

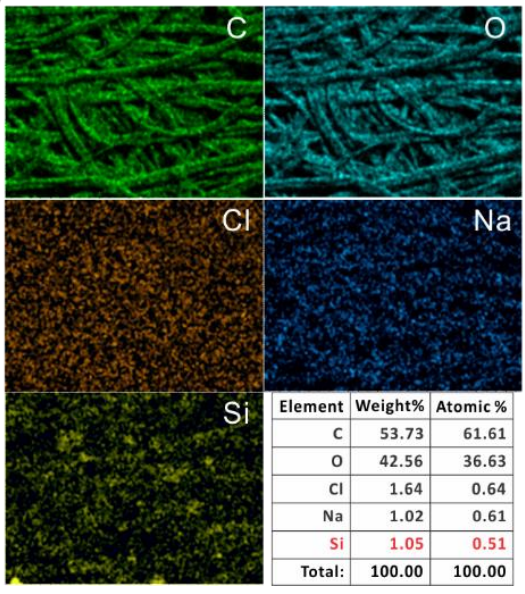

(f)

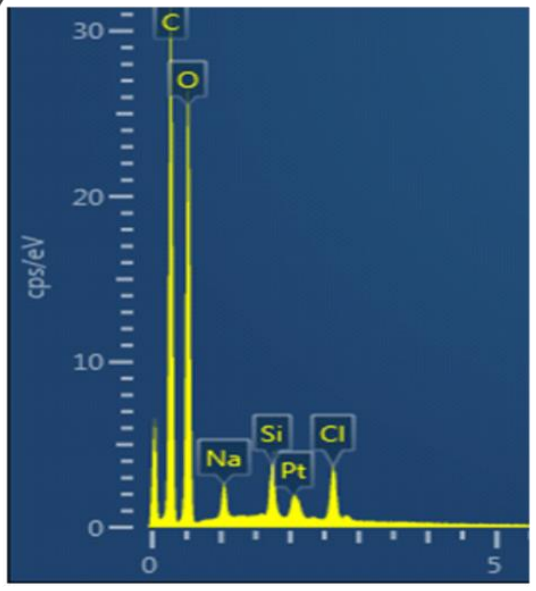

Figure S4. Elemental analysis of COS-treated A4 paper samples with different rinsing steps (a, b,

c) after water rinsing step and (d, e, f) after the hexane rinsing step. (a, b, d, e) Representative SEM images of $(a, d)$ original paper with corresponding elemental mappings and (c, f) elemental spectra. 

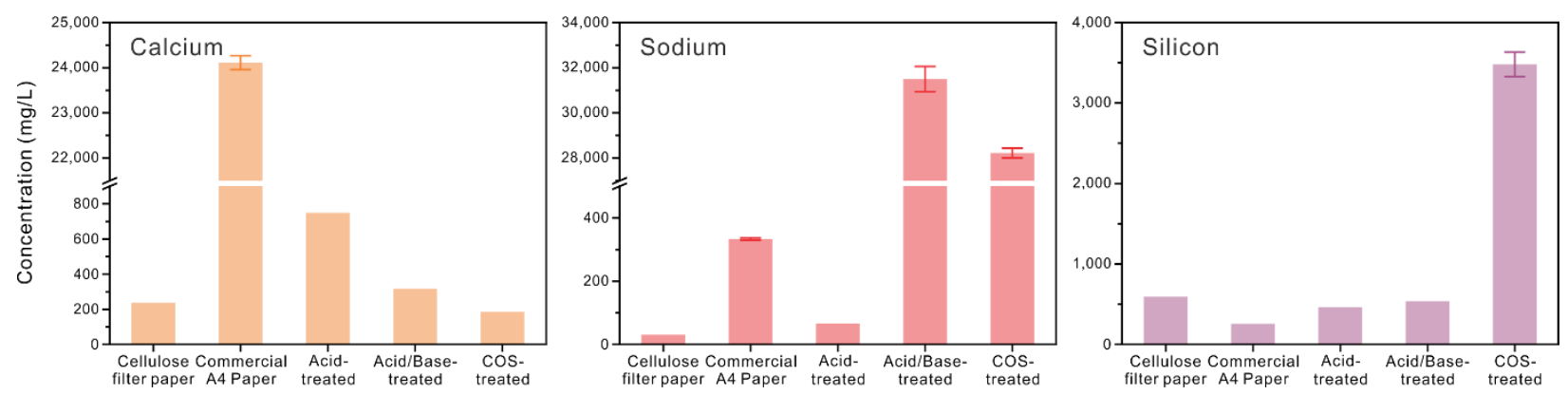

Figure S5. ICP analysis of chemically modified A4 membranes for three different elements: (a) $\mathrm{Ca}$, (b) Na, and (c) Si. Error bars lower than the $1 \%$ range were omitted for clarity.

Table S1. Residual concentration of different elements in membranes prepared in this study based on ICP-MS measurement.

\begin{tabular}{|c|c|c|c|c|c|c|}
\hline \multirow[b]{2}{*}{ Membrane type } & \multicolumn{2}{|c|}{ Calcium } & \multicolumn{2}{|c|}{ Sodium } & \multicolumn{2}{|c|}{ Silicon } \\
\hline & Conc. (mg/L) & $* \operatorname{RSD}(\%)$ & Conc. (mg/L) & RSD (\%) & Conc. (mg/L) & RSD (\%) \\
\hline Cellulose filter paper & 238.7 & 0.7 & 30.6 & 2.8 & 593.8 & 1.9 \\
\hline Commercial A4 paper & 24111.2 & 0.6 & 333.6 & 1.1 & 255.2 & 1.8 \\
\hline Acid-treated paper & 749.3 & 0.4 & 65.5 & 1.7 & 463.6 & 2.2 \\
\hline Acid/base-treated paper & 317.3 & 0.8 & 31499.9 & 1.8 & 535.9 & 2.3 \\
\hline COS-treated paper & 185.0 & 1.1 & 28225.0 & 0.8 & 3480.4 & 4.3 \\
\hline
\end{tabular}

*RSD: Relative standard deviation 

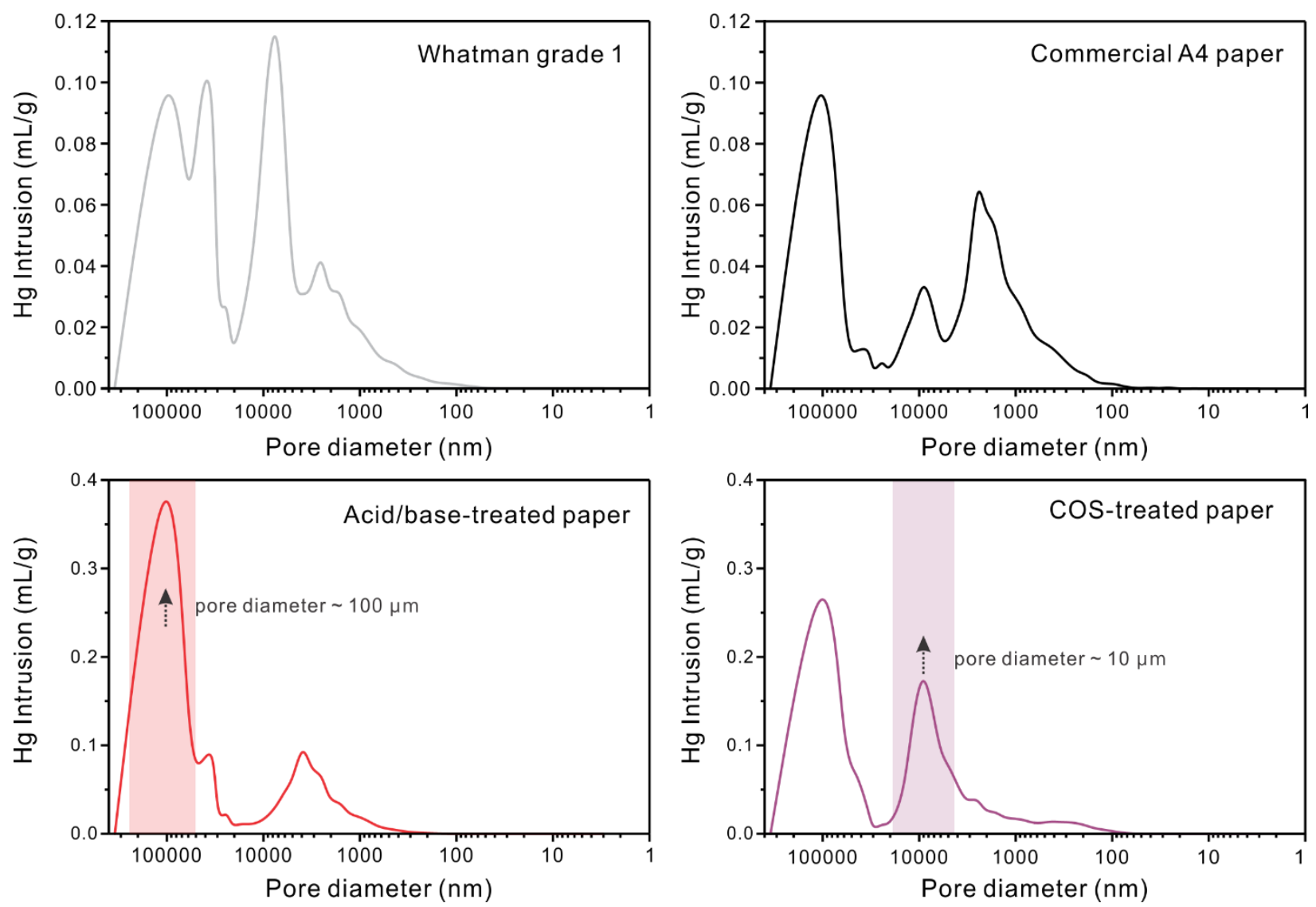

Figure S6. Incremental Hg intrusion $v s$. pore diameter curves obtained from mercury intrusion porosimetry (MIP) tests of specimens: (a) cellulose filter paper, (b) commercial A4 paper, (c) acid/base-treated paper, and (d) COS-treated paper. 
Table S2. Different pore diameters of A4 paper membranes prepared in this study based on capillary flow porosimetry (CFP) measurement. The measurement was measured with five sets per each sample.

\begin{tabular}{|c|c|c|c|c|}
\hline Membrane type & $\begin{array}{c}\text { Minimum } \\
\text { pore diameter }(\boldsymbol{\mu m})\end{array}$ & $\begin{array}{c}\text { Mean } \\
\text { pore diameter }(\boldsymbol{\mu m})\end{array}$ & $\begin{array}{c}\text { Largest } \\
\text { pore diameter }(\boldsymbol{\mu m})\end{array}$ & Error $(\boldsymbol{\%})$ \\
\hline Cellulose filter paper & $0.23 \pm 0.06$ & $2.63 \pm 0.22$ & $15.97 \pm 0.67$ & 3.04 \\
\hline Commercial A4 paper & $0.14 \pm 0.003$ & $1.21 \pm 0.31$ & $4.86 \pm 0.20$ & 1.21 \\
\hline Acid-treated paper & $0.37 \pm 0.07$ & $1.87 \pm 0.03$ & $6.22 \pm 0.16$ & 1.58 \\
\hline Acid/base-treated paper & $0.51 \pm 0.29$ & $1.96 \pm 0.08$ & $6.65 \pm 0.36$ & 2.19 \\
\hline COS-treated paper & $0.49 \pm 0.21$ & $2.17 \pm 0.04$ & $6.71 \pm 0.19$ & 1.71 \\
\hline
\end{tabular}

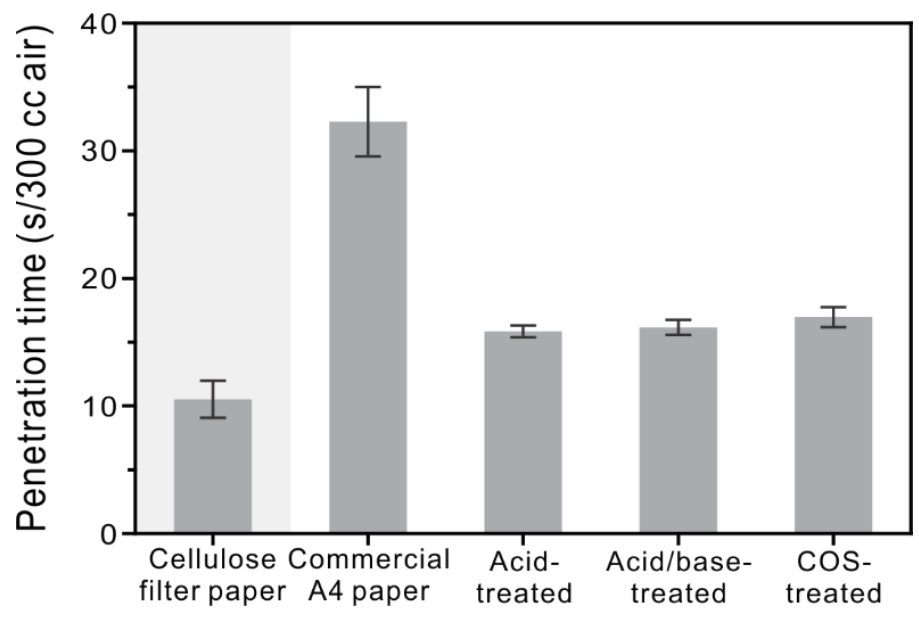

Figure S7. Gurley test results of A4 paper membranes and commercial cellulose filter paper. 
(a)

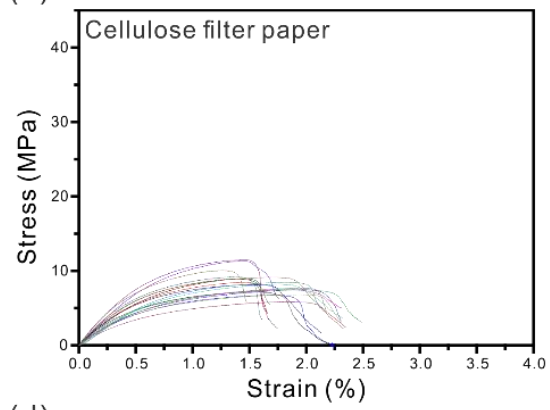

(d)

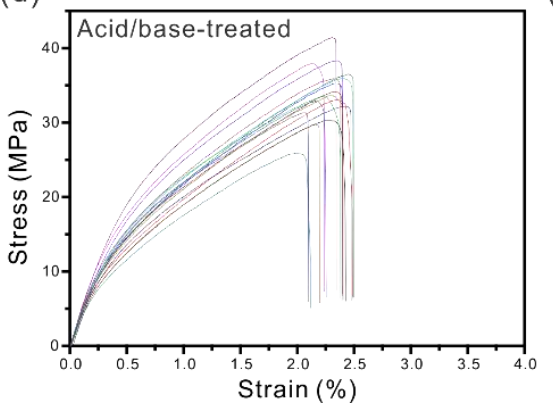

(b)

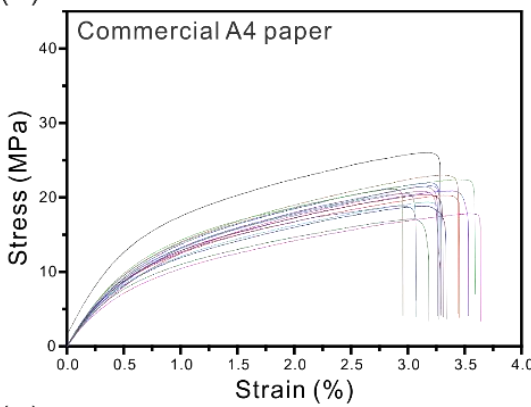

(e)

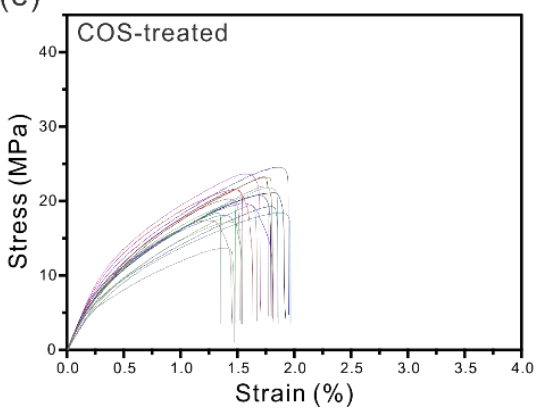

(c)

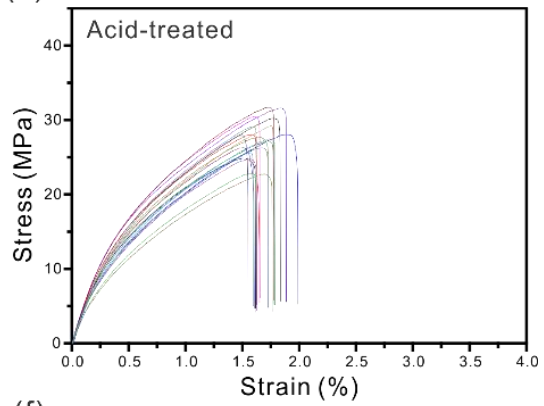

(f)

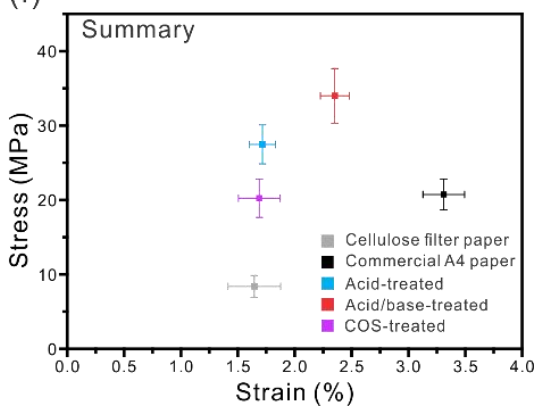

Figure S8. Stress-strain curves of all membranes prepared in this study: (a) cellulose filter paper, (b) commercial A4 paper, (c) acid-treated A4 paper, (d) acid/base-treated A4 paper, (e) COStreated paper, and (f) summary of all prepared membranes.

Table S3. Mechanical properties of all membranes prepared in this study.

\begin{tabular}{|c|c|c|c|}
\hline Membrane type & Strain to fracture (\%) & Ultimate strength (MPa) & Toughness $\left(\mathbf{M J} \mathbf{~ m}^{\mathbf{- 3}}\right)$ \\
\hline Cellulose filter paper & $1.64 \pm 0.23$ & $8.37 \pm 2.57$ & $12.27 \pm 1.75$ \\
\hline Commercial A4 paper & $3.31 \pm 0.18$ & $20.74 \pm 2.09$ & $50.09 \pm 5.64$ \\
\hline Acid-treated paper & $1.71 \pm 0.11$ & $27.47 \pm 2.62$ & $31.25 \pm 4.69$ \\
\hline Acid/base-treated paper & $2.32 \pm 0.12$ & $31.64 \pm 3.50$ & $46.75 \pm 5.80$ \\
\hline COS-treated paper & $1.69 \pm 0.18$ & $8.37 \pm 1.46$ & $24.33 \pm 3.90$ \\
\hline
\end{tabular}



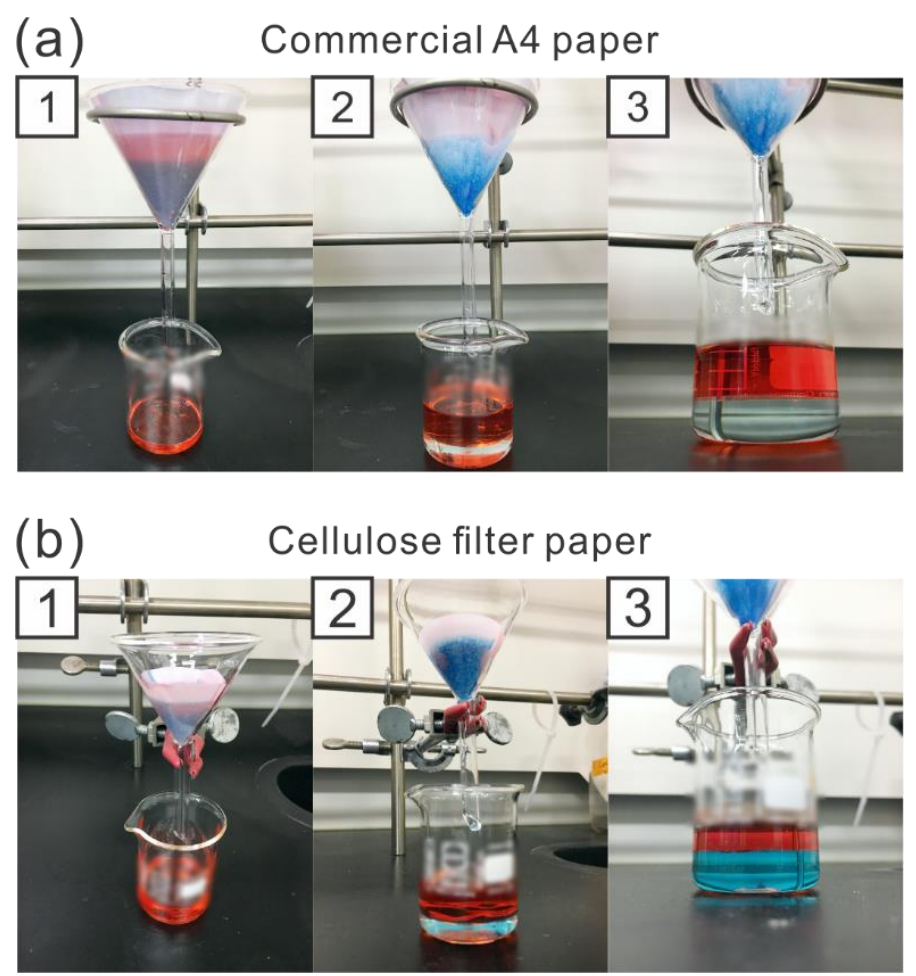

Figure S9. Sequential processes of the water/hexane filtration tests with (a) commercial A4 paper, and (b) cellulose filter paper. The dye concentration was equivalent in both experiments.

(a)

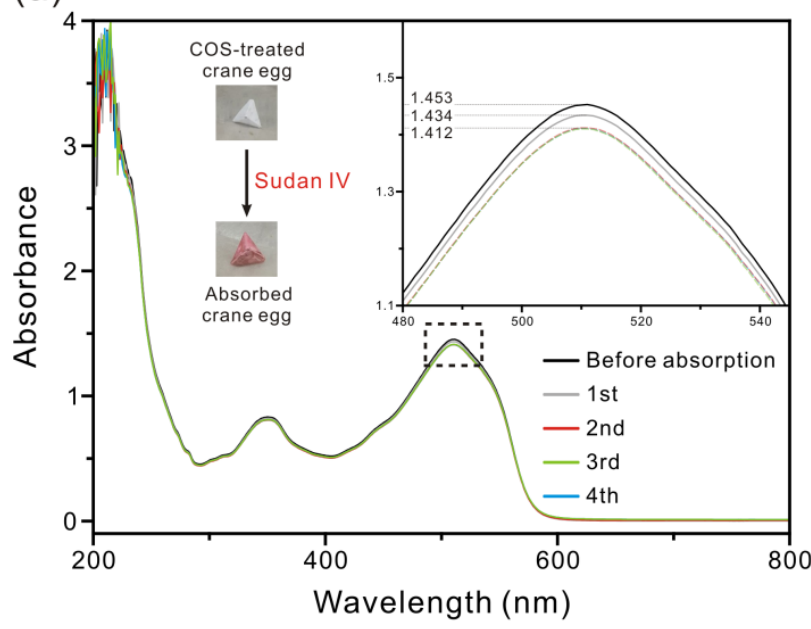

(b)

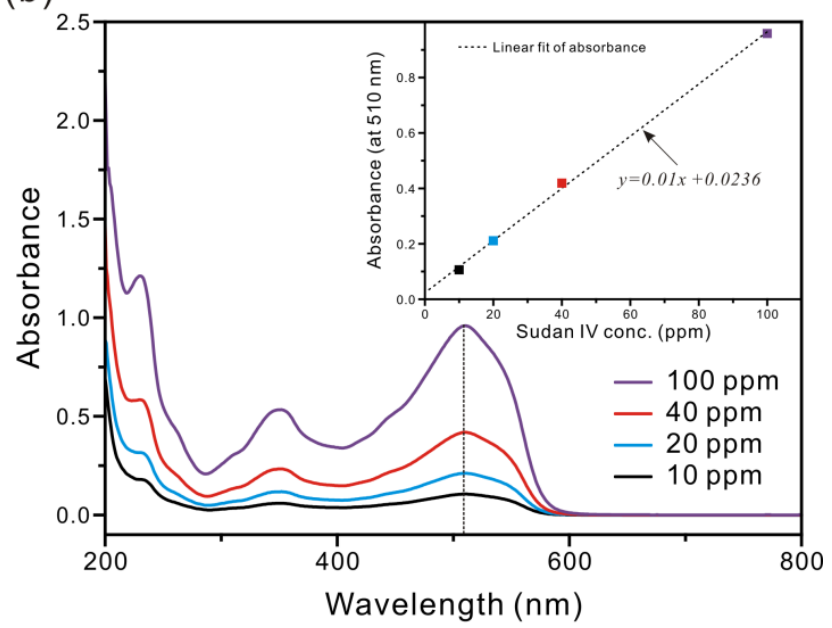

Figure S10. (a) UV-vis spectra of before and after absorption of Sudan IV dye and (b) calibration curve of Sudan IV in hexane. 

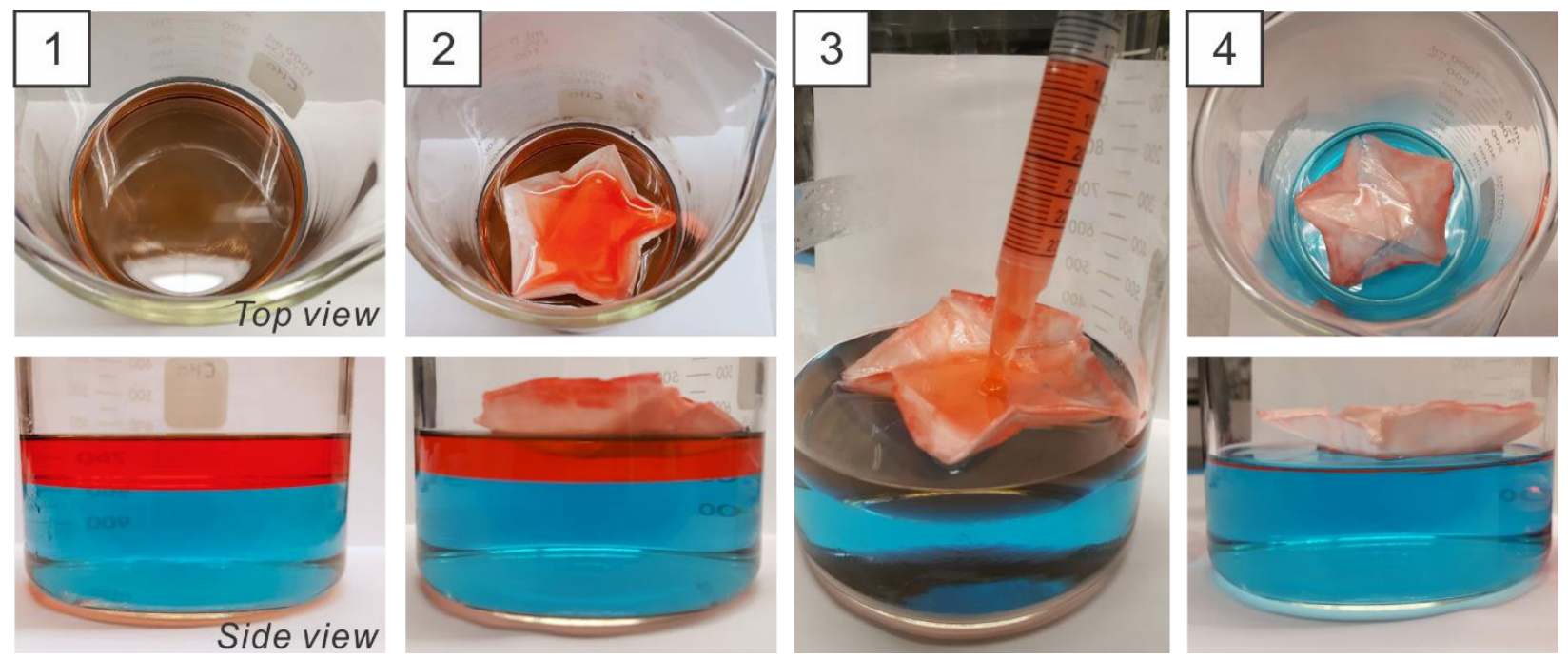

Figure S11. Sequential processes of the selective removal of colored hexane by a star-shaped origami mini boat using COS-treated membrane. 
Supporting Information

\section{REFERENCES}

Energy Inputs for Paper Production from 1965 to 2015. https://www.statista.com/statistics/713287/energy-consumed-by-paper-production/ (accessed March 9, 2020), published by Statista Research Department, March 1, 2016.

(S2) Forest Product Statistics. http://www.fao.org/forestry/statistics/81757/en/ (accessed March 9, 2020), Food and Agriculture Organization of the United Nations

(S3) Distribution of Paper Production Worldwide in 2017 by Grade. https://www.statista.com/statistics/595980/paper-production-distribution-worldwide-by-grade/ (accessed March 9, 2020), published by M. Garside, September 10, 2019. 